\title{
Negative absolute electron mobility, Joule cooling, and the second law
}

\author{
R. E. Robson a) \\ Research School of Physical Sciences and Engineering, Australian National University, Canberra 2600, \\ Australia \\ Z. Lj. Petrović and Z. M. Raspopović \\ Institute of Physics, P.O. Box 68, 11080 Zemun, Belgrade, Yugoslavia \\ D. Loffhagen \\ Institut für Niedertemperatur-Plasmaphysik, 17489 Greifswald, Greifswald, Germany
}

(Received 4 August 2003; accepted 8 September 2003)

\begin{abstract}
A number of recent theoretical investigations of electron motion in attaching gases demonstrate the possibility of a steady-state situation in which the electric current opposes the applied field. This phenomenon, which has been called "negative absolute electron mobility", implies a Joule cooling effect and an associated negative entropy production, suggesting, at first glance, a possible violation of the second law of thermodynamics. In this article we show that the entropy production has in fact two components, the expected negative contribution due to "Joule cooling," and an additional positive part arising from "attachment heating." We insist that the total entropy production be positive, in accordance with the second law, and this has the practical implication that the measurable ("bulk") electron drift velocity must always be positive, even though the actual average ("flux") velocity may be negative. We discuss the phenomenon physically and take as a numerical example electrons in $\mathrm{Ar} / \mathrm{F}_{2}$ mixtures, using Monte Carlo simulation and approximate momentum transfer theory methods to highlight the distinction between the two types of transport coefficient.
\end{abstract}

(C) 2003 American Institute of Physics. [DOI: 10.1063/1.1622667]

\section{INTRODUCTION}

In this article, we discuss some fundamental questions raised by the recent theoretical prediction of the existence of a steady-state current flow opposing the applied electric field. This phenomenon, which so far specifically deals with electrons in certain strongly attaching gases, ${ }^{1,2}$ is sometimes called negative absolute mobility, to distinguish it from timedependent negative transient mobility, which arises for different reasons. ${ }^{3}$ Given that any current flow directed opposite to the applied field has an associated negative entropy production, the obvious first question is: Is the second law of thermodynamics violated? The perennial question of whether a suitably constructed system could violate the second law, ${ }^{4}$ and the associated possibility of making a Maxwell's demon, ${ }^{5}$ continues to greatly interest physicists. Does a simple "swarm" of noninteracting electrons in a bath of attaching neutral gas molecules constitute such a system in certain circumstances? If not, how do we reconcile the predictions with the second law?

The second question is of both fundamental and practical significance: Can such a negative current actually be observed? This answer to this question requires discussion of transport coefficient definition and to considerations of what is actually measured in standard experiments. ${ }^{6}$ Note that we are not talking about subtle differences of a few percent in magnitude, as might be the case in many "swarm" experiments, but rather a difference in the sign of the transport

${ }^{a)}$ Electronic mail: rer105@rsphysse.anu.edu.au coefficient itself. The difference is thus not one of mere esoteric significance.

We shall attempt to answer both these questions together, as simply and straightforwardly as possible, by relying as much as possible upon established results in the literature, both in the general area of nonequilibrium thermodynamics ${ }^{7}$ and in specific discussions of electron transport phenomena in gases. ${ }^{6}$

\section{TRANSPORT THEORY AND ANALYSIS OF A SPECIAL CASE}

\section{A. Bulk and flux drift velocities}

To achieve the desired end we take for simplicity a steady, spatially uniform swarm of electrons in a neutral gas of temperature $T_{0}$ number density $n_{0}$, of infinite extent. There are basically two types of transport coefficients in the presence of collisions which do not conserve number of particles (electrons in this case).$^{6}$ If the applied field $\mathbf{E}=(0,0$, $-E), E>0$ is directed in the $-z$ direction, then electrons of charge $-e$ suffer an acceleration in the $+z$ direction, and eventually acquire an equilibrium average or flux drift velocity

$$
w=\left\langle c_{z}\right\rangle=\frac{1}{n} \int c_{z} f(\mathbf{c}) d \mathbf{c},
$$

where $f(\mathbf{c})$ is the electron velocity distribution function and

$$
n=\int f(\mathbf{c}) d \mathbf{c}
$$


is the electron number density. Normally we find $w>0$, leading to a current density $\mathbf{J}=(0,0,-$ new $)$ directed parallel to E. However, it has been found that if the momentum transfer collision frequency $\nu_{m}(\epsilon)$ increases sufficiently strongly over a range of energies $\epsilon=\frac{1}{2} m c^{2}$ in which there is also a large but sharply decreasing attachment collision frequency $\nu_{a}(\epsilon)$, there is a "window" of electric field strengths, measured by the ratio $E / n_{0}$ (where $n_{0}$ is the gas molecule number density) for which the electrons move "backwards,"

$$
w<0
$$

i.e., the electric current density is now directed opposite to the field direction. Mathematical explanations have been advanced for this phenomenon in terms of "hole burning" in the energy distribution function. ${ }^{2}$ More basic physical arguments ${ }^{1}$ extending those advanced for negative transient mobility $^{3}$ go as follows. Overall, the electrons have their mean energy raised through the strong "attachment heating" effect $^{6,8}$ (preferential loss of low energy electrons due to attachment in this range of $E / n_{0}$ ) but there is a discrimination in preferred direction due to nonattaching collisions: those moving with the field gain further energy and suffer more collisions than those moving against the field, which thus have an ever-decreasing probability of scattering. In other words, attachment pushes the electrons to higher energies, but the combination of field and nonattaching collisions favors backward motion overall, i.e., $w<0$.

There is yet another way of looking at the phenomenon: normally with $w>0$, a steady energy state is achieved by a balance between a power input from the applied field $\mathbf{J} \cdot \mathbf{E}$ $=n e w E>0$ and dissipation of energy by particle-conserving elastic collisions. In the present case, however, such nonattaching collisions are insufficient to dissipate the very strong attachment heating effect, and a steady state can be reached only if $\mathbf{J} \cdot \mathbf{E}=$ new $E<0$ and hence $w<0$. Instead of the usual Joule heating, one might say that there is a "Joule cooling" effect.

Note that inelastic collisions may also play an important role by moderating the attachment heating effect to some extent. These ingredients are all to be found, for example, in $\mathrm{Ar} / \mathrm{F}_{2}$ mixtures (see Fig. 1 of Ref. 2).

On the other hand, standard experiments by and large measure a different transport quantity called the bulk drift velocity $W^{6}$ in the presence of nonconservative collisions. For example, in a time-of-flight experiment, $W$ is simply the velocity of the center-of-mass of the centroid of the pulse, and this is not equal to $w$. The relationship between the two types of drift velocity is well understood in physical terms and can be most simply expressed quantitatively by the approximate relation $^{6}$

$$
W \approx w-\frac{2\langle\epsilon\rangle}{3 e} \frac{d\left\langle\nu_{a}(\epsilon)\right\rangle}{d E} \approx w-\frac{2\langle\epsilon\rangle}{3 e} \frac{d \nu_{a}(\langle\epsilon\rangle)}{d\langle\epsilon\rangle} \frac{d\langle\epsilon\rangle}{d E} .
$$

When the attachment frequency decreases with energy, as is the situation for negative absolute mobility conditions,

$$
\frac{d \nu_{a}(\langle\epsilon\rangle)}{d\langle\epsilon\rangle}<0
$$

TABLE I. Calculation of bulk drift velocity $W$ from flux drift velocity $w$ for electrons in a 0.995/0.005 mixture of $\mathrm{Ar} / \mathrm{F}_{2}$, using Eq. (2) and data (columns 2, 3, and 4) from solution of Boltzmann's equation in the steady homogeneous state. Gas temperature and number density are $300 \mathrm{~K}$ and $2.44 \times 10^{25} \mathrm{~m}^{-3}$, respectively.

\begin{tabular}{ccccc}
\hline \hline$E / n_{0}(\mathrm{Td})$ & $w\left(10^{2} \mathrm{~m} / \mathrm{s}\right)$ & $\langle\epsilon\rangle(\mathrm{eV})$ & $\left\langle\nu_{a}(\epsilon)\right\rangle\left(10^{8} \mathrm{~s}^{-1}\right)$ & $W\left(10^{4} \mathrm{~m} / \mathrm{s}\right)$ \\
\hline 0.06 & -3.8 & 1.14 & 3.1 & 7.6 \\
0.1 & -4.4 & 1.35 & 2.1 & 5.1 \\
0.2 & -4.1 & 1.70 & 1.1 & 1.5 \\
0.5 & -0.9 & 2.20 & 0.74 & 0.7 \\
\hline \hline
\end{tabular}

both physical arguments and Eq. (2) show that the bulk drift velocity is always larger

$$
W>w \text {. }
$$

The question of the sign of $W$ is of course another matter, and obviously depends upon the relative magnitude of the two terms on the right-hand side of Eq. (2).

\section{B. Some numerical results for $\mathrm{Ar} / \mathrm{F}_{2}$}

A comparison of $W$ and $w$ for a $0.995 / 0.005$ mixture of $\mathrm{Ar} / \mathrm{F}_{2}$ at gas temperature of $300 \mathrm{~K}$, based on approximate calculations using Eq. (2), is shown in Table I. The unit of $\mathrm{E} / \mathrm{n}_{0}$ is the townsend $\left(1 \mathrm{Td}=10^{-21} \mathrm{~V} \mathrm{~m}^{2}\right)$. Although the numerical values of $W$ are to be taken as semiquantitative at best, it is clear that while $w$ is negative, the bulk drift velocity $W$ is positive over the entire range of fields, i.e., the second term on the right-hand side of Eq. (2) dominates. The same can be deduced from the time-dependent Monte Carlo simulation, also for a $0.995 / 0.005$ mixture of $\mathrm{Ar} / \mathrm{F}_{2}$ (but at zero gas temperature, and therefore not to be compared quantitatively with Table I) shown in Fig. 1. The procedure for the simulation and the basic benchmarks have been given in Ref. 9. Thinking of a time-of-flight experiment, these numbers indicate that although the electrons are indeed moving backwards $(w<0)$, the "wave" of attachment loss causes the centroid of the pulse to effectively move forward $(W>0) .{ }^{10}$

As is now shown on the basis of nonequilibrium thermodynamics, these appear to be particular examples of a general result.

\section{NONEQUILIBRIUM THERMODYNAMICS AND CALCULATION OF ENTROPY PRODUCTION}

The entropy $s$ per electron is defined by ${ }^{7}$

$$
n s=-k \int f(\ln f-1) d \mathbf{c}=-n k\langle\ln f-1\rangle
$$

and the rate of change due to attaching collisions is

$$
\frac{d n s}{d t}=n k\left\langle\nu_{a}(\epsilon) \ln f(\mathbf{c})\right\rangle \text {. }
$$

Since the electrons are lost according to

$$
\frac{d n}{d t}=-n\left\langle\nu_{a}(\epsilon)\right\rangle
$$




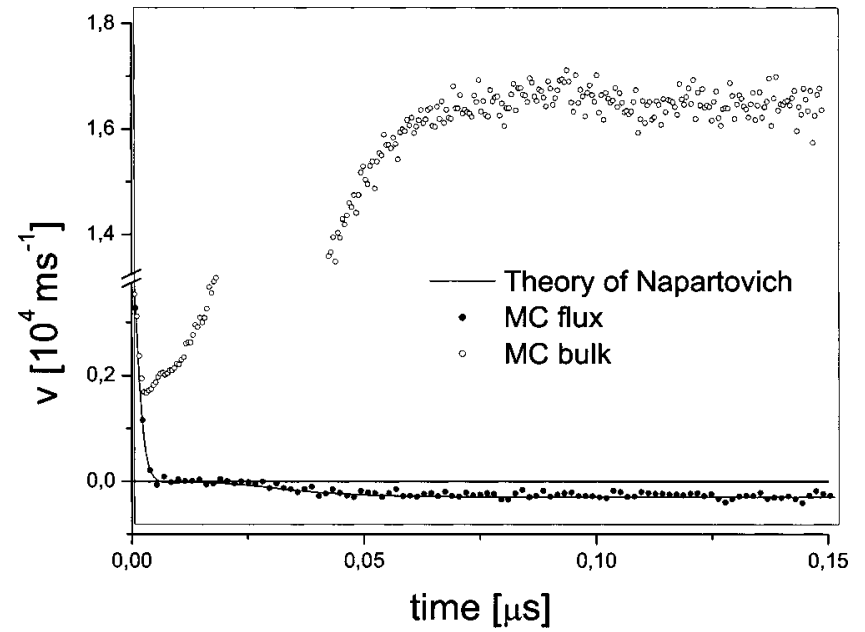

FIG. 1. The time dependence of drift velocities obtained by Monte Carlo simulations from an initial Maxwellian electron distribution function in the mixture $0.995 / 0.005 \mathrm{Ar} / \mathrm{F}_{2}$ for $E / n_{0}=0.1 \mathrm{Td}$ and zero gas temperature. The flux drift velocity $w$ is denoted by closed circles, the bulk drift velocity $W$ by open circles, and the two term theory of Napartovich and co-workers by a thin solid line.

$$
\begin{aligned}
n \frac{d s}{d t} & =n k\left[\left\langle\nu_{a}(\epsilon) \ln f(\mathbf{c})\right\rangle-\left\langle\nu_{a}(\epsilon)\right\rangle\langle\ln f(\mathbf{c})\rangle+\left\langle\nu_{a}(\epsilon)\right\rangle\right] \\
& \equiv \sigma_{a}
\end{aligned}
$$

gives the entropy production per unit time and volume due to attachment. On the other hand the entropy production due to Joule heating ${ }^{7,11}$ is given by

$$
\sigma_{J}=\frac{n e w E}{T},
$$

where $T \approx 2 \epsilon / 3 k$ is the electron temperature. The total entropy production is found simply by adding the two expressions, i.e.,

$$
\begin{aligned}
\sigma=\sigma_{a}+\sigma_{J}= & \frac{n e w E}{T}+n k\left[\left\langle\nu_{a}(\epsilon) \ln f(\mathbf{c})\right\rangle-\left\langle\nu_{a}(\epsilon)\right\rangle\right. \\
& \left.\times\langle\ln f(\mathbf{c})\rangle+\left\langle\nu_{a}(\boldsymbol{\epsilon})\right\rangle\right] .
\end{aligned}
$$

This expression is developed further in the following and forms the basis for all further analysis.

The term in square brackets on the right-hand side of Eq. (8) can be evaluated using the same approximation techniques as in momentum transfer theory, ${ }^{6}$ namely, we expand the attachment frequency in a Taylor series about the mean energy,

$$
\nu_{a}(\epsilon) \approx \nu_{a}(\langle\epsilon\rangle)+(\epsilon-\langle\epsilon\rangle) \nu_{a}^{\prime}(\langle\epsilon\rangle)+\cdots .
$$

Furthermore we must recall that nonequilibrium thermodynamics is strictly speaking valid only for situations not too far from equilibrium, i.e., for weak fields, and consistent with that, we may assume a Maxwellian distribution function in order to evaluate the averages. After some algebra, taking account of the weak field constraint by representing mean energy as a quadratic in $E$, i.e., $\langle\epsilon\rangle=\epsilon_{0}+\epsilon_{1} E^{2}$, and using Eq. (2), the expression for entropy production becomes approximately

$$
\begin{aligned}
\sigma & \approx \frac{n e E}{T}\left(w-\frac{2\langle\epsilon\rangle}{3} \nu_{a}^{\prime}(\langle\epsilon\rangle) \frac{d\langle\epsilon\rangle}{d E}\right)+n k\left\langle\nu_{a}(\epsilon)\right\rangle \\
& =\frac{n e W E}{T}+n k\left\langle\nu_{a}(\epsilon)\right\rangle .
\end{aligned}
$$

If, as has been reasoned before when deriving the generalized Einstein relations from nonequilibrium thermodynamics, ${ }^{7,11}$ the same mathematical form can be assumed to apply for $\sigma$ at high as well as at the low fields, then the result (10) would generally be true. If we now insist that the second law holds, then the entropy production (10) must be positive definite, ${ }^{7}$ i.e., $\sigma>0$. A necessary and sufficient condition for this to be true for arbitrary fields $E$ is

$$
W>0 \text {. }
$$

Thus, by imposing the second law it can be seen that experiments which measure $W$, not $w$, can be expected to yield a positive drift velocity, even under negative mobility conditions. The numerical calculations for $\mathrm{Ar} / \mathrm{F}_{2}$ presented in Sec. II B are just particular examples of this general result.

\section{DISCUSSION}

There are three distinct parts to the above-presented analysis:

(ii) An example using Monte Carlo simulation.

(iii) Nonequilibrium thermodynamics, evaluation of entropy production, and implications of the second law.

Of these, the last provides the incontrovertible evidence for the general result (11), whereas the first two are more in the nature of clues to the final result.

It is clear that the contribution $\sigma_{a}$ of attachment to the overall entropy production (10) is positive and that it must outweigh the negative term $\sigma_{J}$ arising from Joule cooling, in order that the second law be satisfied. An apparent violation of the second law arises only if $\sigma_{a}$ were neglected, and that would be quite incorrect. Question I is therefore answered in the negative. We have simultaneously answered question II with a qualified "no," since the drift velocity measured in standard swarm experiments ${ }^{12}$ is the bulk quantity $W$ which by Eq. (11) is positive under all conditions. Different experiments would have to be devised to measure negative flux drift velocities $w$.

Yet another way of looking at the phenomenon is that low energy attachment selectively removes thermal electrons and thus plays the role of a Maxwell's demon. ${ }^{5}$ However, the price that one pays for that in thermodynamic terms is the additional entropy production due to the demon. The overall effect is associated with the positive bulk velocity $W$, which incorporates the demon's selective heating, and thus naturally the second law is not violated. The situation for other, smaller systems may be quite different, however. ${ }^{4}$

Finally we emphasize that the negative mobility phenomenon $w<0$ discussed here is real. It will be interesting to see if a situation like this with associated Joule cooling can find an application in technology, e.g., perhaps in conversion from one particular form of energy to electrical energy. 


\section{ACKNOWLEDGMENTS}

The support of the Alexander von Humboldt Foundation is gratefully acknowledged. Two of the authors (Z.P. and Z.R.) acknowledge partial support of MNTRS Project No. 1478 and collaboration and useful discussions on negative mobility with A. Napartovich, N. Dyatko, S. Sakadžić, and S. Dujko. Special thanks goes to Professor R. W. Crompton for stimulating discussions.

${ }^{1}$ N. A. Dyatko, A. P. Napartovich, S. Sakadžić, Z. Lj. Petrović, and Z. Raspopović, J. Phys. D 33, 375 (2000); see also N. A. Dyatko and A. P. Napartovich, Proceedings of the International Symposium on ElectronMolecule Collisions and Swarms, Lincoln, NE, July, 2001, edited by I. Fabrikant, G. Gallup, and P. Burrow.

${ }^{2}$ N. Dyatko, D. Loffhagen, A. P. Napartovich, and R. Winkler, Plasma Chem. Plasma Process. 21, 421 (2001).

${ }^{3}$ B. Shizgal and D. R. A. McMahon, Phys. Rev. A 32, 3699 (1985).

${ }^{4}$ Violations of the second law have recently been observed for few body systems on short time scales by G. M. Wang et al., Phys. Rev. Lett. 89, 050601 (2002)
${ }^{5}$ C. M. Caves, Phys. Rev. Lett. 64, 2111 (1990); W. H. Zurek, Nature (London) 341, 119 (1989)

${ }^{6}$ R. E. Robson, J. Chem. Phys. 85, 4486 (1986); R. E. Robson, Aust. J. Phys. 44, 685 (1991). The distinction between the two types of transport coefficients was first made by H. Tagashira, Y. Sakai, and S. Sakamoto, J. Phys. D 10, 1051 (1977), although their labeling followed the particular, rather than the generic type of experiment. For a recent review of transport theory see R. D. White, K. F. Ness, and R. E. Robson, Appl. Surf. Sci. 192, 26 (2002).

${ }^{7}$ S. R. de Groot and P. Mazur, Non-equilibrium Thermodynamics (NorthHolland, Amsterdam, 1962).

${ }^{8}$ For a recent discussion, see S. Dujko, Z. Lj. Petrović, Z. M. Raspopović, and T. Makabe, International Symposium on Electron-Molecule Collisions and Swarms, Lincoln, NE, July, 2001, pp. 124 and 125.

${ }^{9}$ Z. M. Raspopović, S. Sakadžić, S. Bzenić, and Z. Lj. Petrović, IEEE Trans. Plasma Sci. 27, 1241 (1999).

${ }^{10}$ The direct indication of this effect was given by Monte Carlo simulations of S. Dujko, Z. M. Raspopović, and Z. Lj. Petrović (unpublished).

${ }^{11}$ R. E. Robson, Aust. J. Phys. 25, 685 (1972).

${ }^{12}$ L. G. H. Huxley and R. W. Crompton, The Diffusion and Drift of Electrons in Gases (Wiley, New York, 1974). 